\title{
Новый масштаб обменной энергии квантово-холловских ферромагнетиков \\ Ваньков А.Б., Кукушкин И.В.
}

ИФТТ РАН, 142432, МО, г. Черноголовка, ул. Ак. Осипьяна, 2

DOI 10.34077/Semicond2019-202

Некоторые секреты физики конденсированного состояния раскрываются на примере определенных модельных задач. Так, эффекты, связанные с обменным взаимодействием двумерных электронов в квантующем магнитном поле, изучают в простейшем случае квантово-холловского ферромагнетика (QHF) с $v=1$. Это состояние устойчиво даже в системах со сколь угодно малым Зеемановским взаимодействием, что объясняется значительным выигрышем в обменной энергии. Сведенный до минимума набор встроенных корреляций делает это состояние наиболее пригодным для теоретического анализа, хотя последовательный учет многочастичных эффектов возможен лишь при условии малости кулоновских вкладов по сравнению с циклотронной энергией. Ранее на примере двумерных электронных систем в GaAs был показан эффективный способ зондирования обменных эффектов через энергию циклотронных возбуждений с переворотом спина (CSFE), измеряемую методом неупругого рассеяния света [1]. Наблюдалось хорошее согласие эксперимента с расчетами в приближении Хартри-Фока при выполнении условия малости кулоновских вкладов относительно циклотронной энергии. В появившихся сравнительно недавно системах на основе гетероперехода $\mathrm{ZnO} / \mathrm{MgZnO}$ взаимодействие на порядок сильнее, а сильное смешивание уровней Ландау качественным образом затрудняет теоретическое описание коллективных эффектов, так как даже точный вид основного состояния системы неизвестен. В этих условиях было снова проведено экспериментальное исследование обменной энергии рассеянием света на коллективном возбуждении CSFE.

В настоящей работе было установлено, что в широком диапазоне электронных концентраций, соответствующих параметру Вигнера-Зейтса $7<r_{s}<12$, обменный вклад в энергию CSFE имеет масштаб циклотронной энергии вместо обычной величины кулоновской энергии на расстоянии магнитной длины $e^{2} / \varepsilon \ell_{B}$. При этом прочие свойства коллективного возбуждения - зависимость энергии от фактора заполнения, от температуры, дисперсия от обобщенного импульса - сохраняются. Причиной нетривиального поведения обменного вклада является сильное смешивание уровней Ландау, приводящее к эффективной перенормировке кулоновского взаимодействия, ожидаемого в теории в пределе $r_{s}>>1$. В работе показано, что хорошую оценку многочастичного вклада в энергию коллективных возбуждений можно получить даже в этом пределе, проводя вычисления все же в проекции на несколько нижайших уровней Ландау, но заменяя влияние остальных уровней введением статической диэлектрической функции в Фурье-компоненту Кулоновского потенциала. Вычисления как методом Хартри-Фока, так и методом точной диагонализации дают хорошее согласие с экспериментом и качественно совпадают с альтернативными теоретическими оценками обменной энергии QHF, полученными для задачи о скирмионных возбуждениях [2].

[1] A.B. Van'kov, L.V. Kulik, I.V. Kukushkin et al., Phys. Rev. Lett. 97, 246801 (2006).

[2] S. Dickmann, Phys. Rev. B, 65, 195310 (2002); S. V. Iordanski and A. Kashuba, Pis'ma v ZhETF, 75(7),419; A. P. Smith, A. H. MacDonald, G. Gumbs, Phys.Rev. B 45, 8829 (1992). 